between nerves and muscles induces the accumulation of $\mathrm{ACh}$ receptors (Fischbach, Harvard University) and a low molecular weight substance from chick spinal cord and brain that produces an increase in $\mathrm{ACh}$ receptors of cultured muscle has been extracted. The implication of this and other similar studies is that such substances may play a role in the subsynaptic accumulation of $\mathrm{ACh}$ receptors at neuromuscular junctions.

Reverse trophic influences of the innervated target cells on neurons within the chick ciliary ganglion were described by Landmesser (Yale University) \& Pilar, (University of Connecticut). During development, an excessive number of nerve cells compete to innervate a limited number of target cells; those neurons that form connections are able to survive and mature, while those that fail to connect undergo cell death. The proportion of cells that die can be greatly increased by ablating the target and conversely, the proportion of survivors can be increased by pruning out some of the ciliary neurons. The same effect is also seen in tissue culture; the maturation and survival of ciliary neurons is enhanced by co-culture with an appropriate target tissue, skeletal muscle.

The regulation of nerve sprouting was analyzed by Pestronk and Drachman. Pharmacological denervation (using blockers of nerve conduction of $\mathrm{ACh}$ release) induces sprouting of nerve terminals which branch and enlarge over the surface of each muscle fiber. Extrajunctional ACh receptors appear to play a role in this type of sprouting, since blockade by $\alpha$-BuTx prevents the formation of terminal sprouts. However, in collateral sprouting occurring when neighboring muscle fibers are denervated little effect is produced by blockade of $\mathrm{ACh}$ receptors. Finally, intrinsic factors such as age may greatly effect the nerve's ability to sprout - both terminal sprouting and regeneration after injury are progressively impaired with increasing age.

The basement membrane of the motor endplate may be particularly important in neuromuscular junction formation (McMahan, Harvard University) Even after destruction of skeletal muscle cells, the basement membrane retains its organization, and is capable of serving as a target for regenerating motor nerves. Moreover, the basement membrane appears to act as a scaffold for the reorganization of regenerating muscle. If a muscle is damaged and then allowed to regenerate, new endplates form at their previous sites, as directed by the remaining basement membrane.

Extracellular matrix may also play a role in interactions between Schwann cells and axons. The 'dystrophic' mouse shows a defect in myelination of nerve roots, with some Schwann cells apparently refusing to ensheath the naked axons. Aguayo (Montreal General Hospital) showed that grafting segments of unmyelinated nerve roots from 'dystrophic' mice to normal or even 'dystrophic' mice resulted in correction of the myelination defect. Bunge (Washington University) was able to reproduce the myelination defect in tissue culture of 'dystrophic' mouse dorsal root ganglia, and to correct the abnormality by the addition of fibroblasts or of serum. This suggests that the lack of an extracellular substance normally secreted by Schwann cells results in impaired myelination.

Perhaps the greatest promise for future research in neuromuscular disease lies in the application of a variety of powerful new biological techniques to the eminently workable nerve-muscle system. Among the new techniques described at the conference were monoclonal antibodies to serve as probes of surface membrane components of chick skeletal muscle (Fambrough, Carnegie Institute of Washington) and tissue culture of pathological muscle. Successful culture of skeletal muscle from patients with myotonic dystrophy was demonstrated by Appel (Baylor College of Medicine) and abnormalities in cultured Schwann cells from patients with adrenal leukodystrophy and Issac's syndrome by Askanas (NIH). Finally, the techniques of modern biochemical genetics are just beginning to be applied to skeletal muscle. Recombinant techniques have been used to clone genes for contractile proteins by Yaffe (Weizmann Institute) and Schwartz (Baylor College of Medicine). H. Epstein (Baylor College of Medicine) pointed out the encouraging prospects for identifying the genetic abnormalities in the muscular dystrophies by such methods.

\title{
Crystallizing membrane proteins
} from Richard Henderson

After many years of disappointing failures the first succesful attempts to crystallize membrane proteins have recently been reported. Large threedimensional crystals suitable for X-ray analysis are needed for the resolution of the protein structure in atomic and chemical detail, a level of analysis essential for the understanding of phenomena such as channel selectivity and the mechanism of action of membrane bound pumps and receptors.

In the first paper, Ozawa et al. (Proc. natn. Acad. Sci. U.S.A. 77, 928; 1980) describe crystals of the cytochrome c oxidasecytochrome c complex. These crystals were made from detergent (cholate)-solubilised oxidase almost completely depleted of lipid $(<1 \%)$. Cytochrome $\mathrm{c}$ was added to make a 1:1 complex and crystals obtained by removal of detergent by dialysis. The crystals contained both oxidase and cytochrome $c$ in a 1:1 ratio, but both detergent and lipid were absent. They are thus well-characterised chemically but the extent of crystalline order remains to be determined by X-ray diffraction. At the moment, they are needles rather too small for analysis.

The most successful and promising reports used the detergent, octylglucoside, recently made commercially available. In two exciting reports, crystals were made by precipitation of membrane proteins solubilised in octyl glucoside without removal of the detergent. The crystals thus contained a substantial amount of detergent in addition to aqueous regions, the protein and perhaps also some lipid.

Michel and Oesterhelt (Proc. natn. Acad. Sci. U.S. A. 77, 1283; 1980) used 2.5

Richard Henderson is in the MRC Laboratory of Molecular Biology, University Medical School, Cambridge.
$-2.8 \mathrm{M}$ salt to precipitate octyl glucosidesolubilised bacteriorhodopsin, obtaining two crystal forms, one needle-shaped and the other cube-shaped. These crystals are truly three-dimensional and one form (the needle) is ordered to at least $8 \AA$ resolution. The needles have the interesting property of being strongly dichroic and the purple chromophore is oriented almost exactly along the needle axis. Further structural work should therefore be useful to determine the chromophore orientation and if better diffraction patterns can be obtained, the crystals may well be suitable for higher resolution analysis.

Garavito and Rosenbusch (J. Cell Biol. 86,$327 ; 1980$ ), used polyethylene glycol to precipitate the pore-forming protein, porin that comes from $E$. coli. outer membranes, after solubilising with octyl glucoside. They obtained birefringent rhombic plates and prisms using $\beta$-octyl glucoside and non-birefringent but larger, bipyramids using $\alpha$-octyl glucoside which they had to synthesize themselves. The large bipyramids gave X-ray diffraction spots out to $3.8 \AA$ resolution making them ordered at least to a resolution close to that required to reveal atomic detail.

This recent flurry of successful crystallizations may now mean that membrane proteins are sufficiently purified and characterised, and that detergent development is sufficiently well-advanced, that a substantial effort to crystallize many more membrane proteins should be started. Perhaps these results, exciting in themselves, will provide the impetus for these efforts. It seems that it would now be worthwhile both to try to crystallize other membrane proteins and to develop other detergents like octyl-glucoside, which itself remains in solution while the proteindetergent complex is precipitated. 